\title{
Review: counselling in primary care may have short term benefits for people with common mental disorders
}

\author{
Bower P, Rowland N, Hardy R. The clinical effectiveness of counselling in primary care: a systematic review and
} meta-analysis. Psychological Med 2003 Feb;33:203-15.

\section{QUESTION: Is counselling people with common mental disorders in primary care more effective than usual general practitioner care or alternative mental health treatments?}

\section{Design}

Systematic review with meta-analysis.

\section{Data sources}

The reviewers searched Medline, Embase, AMED, ASSIA, HealthSTAR, DHSS DATA, DARE, NHSeed, HELMIS, EconLit, CINAHL, PsycLit, the Cochrane Controlled Trials Register and the Cochrane Collaboration on Depression, Anxiety and Neurosis (CCDAN) Trials Register to 2003. The reviewers hand-searched a specialist journal, scanned reference lists, and approached experts for additional papers.

\section{Study selection}

Randomised controlled trials and controlled clinical trials were eligible if they compared people with psychological or psychosocial problems receiving counselling in primary care with a control or comparison condition (usual GP care or treatments such as medication or cognitive behavioural therapy). Only trials in which counsellors were trained to British Association for Counselling and Psychotherapy accreditation levels or equivalent were included. There were no language restrictions.

\section{Data extraction}

Two reviewers selected studies and extracted data independently. Disagreements were resolved by discussion. The reviewers used a standardised rating system to assess internal and external validity. Meta-analytic techniques were used to pool data on the effect of counselling on anxiety and depression symptoms. The authors conducted sensitivity analyses to test the robustness of the findings.

\section{Main results}

The authors included 7 studies, with follow up ranging from 6 weeks to 12 months. Changes in anxiety and depression were the main outcome measure, assessed using the Beck Depression Inventory, Hospital Anxiety and Depression Scale, Symptom Inventory, and General Health Questionnaire, amongst others. "Short term" and "long term" outcomes were measured, although the authors do not describe the length of these periods.

Counselling in general practice reduced symptoms of depression and anxiety over usual general practitioner care in the short term (standardised mean difference $-0.28,95 \%$ CI -0.43 to $-0.13, \mathrm{n}=741$ in 6 trials). This difference did not hold in the longer term (standardised mean difference $-0.07,95 \%$ CI -0.26 to $0.12, n=447$ in 4 trials, "long term" timeframe unclear).

One trial compared counselling with antidepressant treatment administered by general practitioners. There were no significant differences in short or long term outcomes. One trial compared counselling with cognitive behaviour therapy. There were no significant differences in short or long term outcomes.

\section{Conclusions}

Compared with usual general practitioner care, primary care counselling may be associated with modest improvements in short term outcomes for people with common mental disorders. There is little comparative data about treatments such as antidepressants or cognitive behaviour therapy, although preliminary data suggest no differences between these treatments and primary care counselling.

\section{COMMENTARY}

Counselling in primary care is, almost by definition, likely to be a non-standardised intervention delivered by clinicians with different skills working under varying conditions. Assessing its impact is complicated because of the wide range of variables that need to be considered. In this review, the authors have done an impressive standardised analysis of previous studies, although their strict criteria meant that only 7 controlled studies were included. Even in these studies, there was still significant variation in entry criteria (degree of distress or diagnosis), counselling approach, and the background of counsellors. None of the studies controlled for physician characteristics. The authors acknowledge difficulties developing more rigorous studies within clinical settings given the need to balance internal validity with external demands (the reality of family practice).

The conclusion that counselling in primary care may have short term benefits is important, although the finding of no change in social functioning, albeit drawn from a single study, requires further exploration. This also raises the question of a "reasonable" timeframe in which to measure the success of a primary care counselling intervention. Is short term improvement sufficient, even if not maintained? Does this reflect the natural course of some of these problems? Or is there a need for a different approach to primary care counselling, with opportunities for periodic follow up sessions?

In order to answer these and similar questions, it may be necessary to broaden our research focus to examine other variables that can contribute to the success of an intervention, especially those related to the process of care. These include the time between symptom onset and presentation to the general practitioner or referral to the counsellor; waiting time for treatment after referral; previous treatment; previous episodes and outcomes; and the relationship between the counsellor and referring physician. We also need to pay greater attention to screening or treatment performed by the family physician prior to referral, the GP's interest and expertise in counselling, and the reason for referral. Are referrals driven by symptom severity or is priority given to cases that are seen as hard to treat, time consuming or where there is a breakdown in the patientdoctor relationship? All of these factors might have an impact on who is referred and their long and short term outcomes.

Professor Nick Kates, MBBS FRCP

Department of Psychiatry and Behavioural Neurosciences McMaster University, Canada
Source of funding: not specified.

For correspondence: P Bower, National Primary Care Research and Development Centre. University of Manchester, United Kingdom. 